\title{
Heterogeneous integration and precise alignment of InP-based photonic crystal lasers to complementary metal-oxide semiconductor fabricated silicon-on-insulator wire waveguides
}

\author{
T. J. Karle, ${ }^{1, a)}$ Y. Halioua ${ }^{1, b)}$ F. Raineri, ${ }^{1, c)}$ P. Monnier, ${ }^{1}$ R. Braive, ${ }^{1, c)}$ L. Le Gratiet, ${ }^{1}$ \\ G. Beaudoin, ${ }^{1}$ I. Sagnes, ${ }^{1}$ G. Roelkens, ${ }^{2}$ F. van Laere, ${ }^{2}$ D. Van Thourhout, ${ }^{2}$ and R. Raj $^{1}$ \\ ${ }^{1}$ Laboratoire de Photonique et de Nanostructures, CNRS UPR20, 91460 Marcoussis, France \\ ${ }^{2}$ Department of Information Technology, Ghent University-IMEC, Sint-Pietersnieuwstraat 41, 9000 Gent, \\ Belgium
}

(Received 13 November 2009; accepted 19 January 2010; published online 18 March 2010)

\begin{abstract}
The integration of two-dimensional III-V InP-based photonic crystal and silicon wire waveguides is achieved through an accurate alignment of the two optical levels using mix-and-match deep ultraviolet (DUV)/electron beam lithography. The adhesively bonded structures exhibit an enhancement of light emission at frequencies where low group velocity modes of the photonic crystal line defect waveguides occur. Pulsed laser operation is obtained from these modes at room temperature under optical pumping. The laser light is coupled out of the $\mathrm{Si}$ waveguide via grating couplers directly to single mode fiber. (C) 2010 American Institute of Physics.
\end{abstract}

[doi:10.1063/1.3319667]

\section{INTRODUCTION}

In the quest for efficiency, integrated optics has recently turned its attention to development of subwavelength, high contrast photonic components. Preferably based upon a silicon platform, these would allow one to envision low-cost and high component density. ${ }^{1}$ To achieve these ambitious goals $\mathrm{Si}$ technology takes advantage of processing knowhow from the electronics industry. Mature complementary metal-oxide semiconductor (CMOS) processing technology renders silicon unsurpassed in terms of fabricated device quality. Furthermore, the ability of low-loss $\mathrm{Si}$ wire waveguides to be bent with tight radii of curvature predisposes them to small device footprints and thus large scale integration. Beyond purely passive features such as guiding and filtering, the inclusion of active components in the $\mathrm{Si}$ platform would vastly enhance the portfolio of optical functions. For this we require devices capable of emitting, modulating, and detecting light, ideally with low power expenditure. Heterogeneous integration of III-V materials onto silicon could offer an efficient solution, providing tailormade optoelectronic properties. A wide variety of materials can be bonded, to allow mixing and matching of different functionalities. ${ }^{2}$ Using this technology Fabry-Perot, distributed feedback and mode-locked lasers have been integrated with silicon-on-insulator (SOI) rib waveguides and integrated microdisk lasers coupled to SOI wire waveguides have been demonstrated. ${ }^{3}$ Patterning this active material on the wavelength scale also permits control of its photonic properties. In this context two-dimensional (2D) photonic crystals (PhCs) allow us to achieve ultrasmall components and can be configured to substantially augment the light-

\footnotetext{
${ }^{a)}$ Electronic mail: Timothy.Karle@lpn.cnrs.fr.

${ }^{b)}$ Also at Ghent University-IMEC, Department of Information TechnologySint-Pietersnieuwstraat 41, 9000 Gent, Belgium.

${ }^{c)}$ Also at Université Paris 7 Denis Diderot, Paris, France.
}

matter interaction, thus reducing laser thresholds and switching energies. ${ }^{4}$ Indeed carrier nonlinearities in patterned III-V materials, [more specifically InP-based Quantum Wells (QWs) Refs. 5 and 6], have larger magnitudes and faster response times compared to silicon. Monolithic integration of active PhC structures with passive waveguides has been investigated using regrowth of passive material ${ }^{7}$ and also intermixing to produce all-InP-based alternatives. ${ }^{8}$ In terms of $\mathrm{PhCs}$ which have been integrated onto $\mathrm{Si}$, in Ref. 9 high contrast modulation was achieved using a benzocyclobutene (BCB) bonded InP-based surface emitting PhC. Similar graphite lattice $\mathrm{PhC}$ structures were also integrated onto a $\mathrm{Si}$ wafer using AuIn eutectic bonding, and continuous wave (CW) laser operation ${ }^{10}$ was then established due to the fivefold higher thermal conductivity of the $\mathrm{SiO}_{2}$ cladding. Rapid modulation of these $\mathrm{PhC}$ lasers integrated on $\mathrm{Si}$ was demonstrated by dynamic measurements of the pulse turn on/off. ${ }^{11}$ These demonstrations point the way to all-guided hybrid systems, which is our concern here. The enhancement of silicon photonics by III-V PhC based optical functions would make for a versatile integrated photonics platform.

In this work, we study the integration of a $\mathrm{PhC}$ membrane containing InGaAsP/InGaAs QW material and SOI wire waveguides, using die-to-die bonding (as shown schematically in Fig. 1). For this heterogeneous integration, we choose adhesive bonding as it is a rapid, convenient alternative to molecular wafer bonding. ${ }^{12}$ It is desirable that the high resolution $\mathrm{PhC}$ patterning that we subsequently apply to the InP slab has a close spatial registration with the underlying $\mathrm{Si}$ waveguide.

In Sec. II, we discuss the proposed hybrid platform consisting of InP-based membrane material bonded; patterned with a $2 \mathrm{D} \mathrm{PhC}$ structure; and coupled to SOI photonic wires. In Sec. III, the technological procedure for the fabrication of the hybrid structures is detailed. Sec. IV shows the measurements of the alignment accuracy between the two optical 


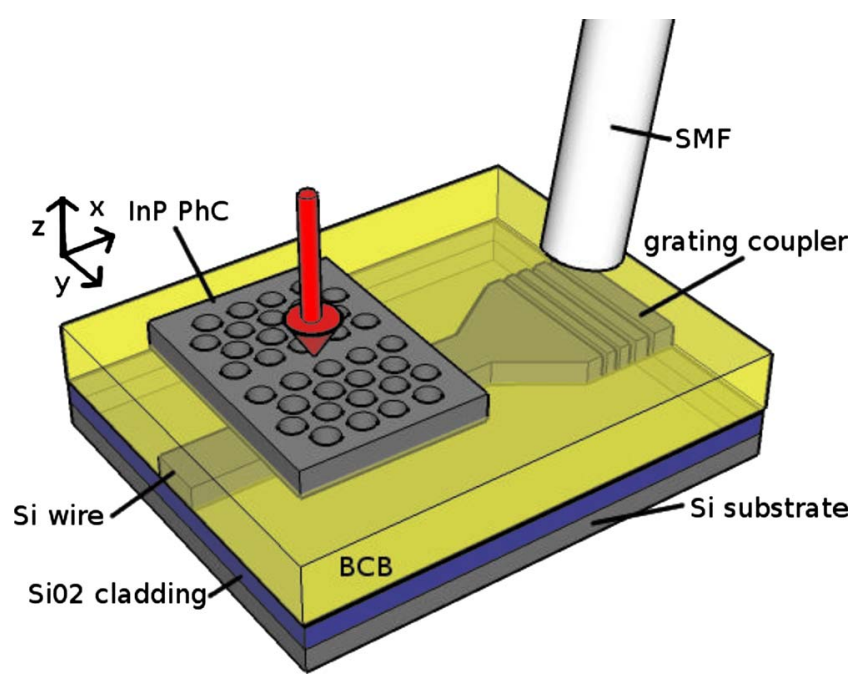

FIG. 1. (Color online) Hybrid III-V PhC/SOI wire structure and experimental configuration. Our PhC defect waveguide hybrid structure is optically surface pumped, as indicated by the red arrow. The underlying Si wire waveguide captures the laser emission which is then diffracted by a grating coupler and collected by a cleaved SMF.

levels of our structures. Finally, in Sec. V we report the optical measurements of the device emission. The luminescence experiments are compared to numerical simulation and results on laser emission are presented.

\section{HYBRID PLATFORM: III-V 2D PHC BONDED AND COUPLED TO SOI WIRE}

SOI photonics takes advantage of silicon's natural oxide as a waveguide cladding. Wire waveguides with cross sectional dimensions below approximately 220 high by $500 \mathrm{~nm}$ wide, provide tight confinement of the optical field and can be seen [in Fig. 2(a)] to be single mode (per polarization). We denote the polarization of the wire modes following the dominant components of their fields, based upon those of the
SOI slab waveguide. TE-like modes are dominated by components of the electric field in the plane of the slab and TM-like with the magnetic field in the plane of the slab. With asymmetry in the vertical direction the wire modes are hybrid, and so here we only loosely apply the terms TE/TMlike, to indicate the dominant polarization.

$\mathrm{PhCs}$ are high contrast periodic dielectric structures, which promote strong interference between forward and backward traveling optical waves. Operating at either the band edge of a uniform $\mathrm{PhC}$ lattice or at the mode edge of a defect waveguide mode gives control over the spatial localization of the optical field and concurrently, control of its group velocity. ${ }^{13}$ We choose a defect waveguide configuration, which is obtained by suppressing one row of air holes in the triangular PhC lattice (W1), in order to provide a system, which is ostensibly monomode (one mode per polarization per waveguide) and highly confined.

In Fig. 2(b) the three-dimensional (3D) band structure of a W1 waveguide is plotted, calculated using a guided mode expansion along the $\Gamma-\mathrm{K}$ direction. ${ }^{14}$ Modes of both polarizations (TE-like and TM-like) are plotted and these are displayed in blue for odd and in red for even lateral symmetry. Mirror reflection with respect to the vertical plane $(\mathrm{x}-\mathrm{z})$ is a symmetry operation of the waveguide. The modes are classified as even $\left(\sigma_{\mathrm{xz}}=+1\right)$ or odd $\left(\sigma_{\mathrm{xz}}=-1\right)$ with respect to this mirror plane. The dispersion of the odd TE-like defect mode close to $(\mathrm{k}=\pi / \mathrm{a}, \mathrm{u}=\mathrm{a} / \lambda=0.275)$, is especially flat. In this low $\mathrm{v}_{\mathrm{g}}$ regime the interaction between the matter and the optical field can be greatly enhanced. ${ }^{15}$ We would like to interact efficiently with the guided $\mathrm{PhC}$ modes in the window $\mathrm{k}_{\mathrm{x}}=0.45-0.55 \times 2 \pi / \mathrm{a}$ for normalized frequencies, $\mathrm{u}$ $=0.275-0.3$, beneath the BCB light line. This is a regime that previous waveguide- $\mathrm{PhC}$ coupling schemes have been unable to attain due to their lower effective waveguide mode indices. ${ }^{16}$ We therefore opt for a nominal SOI wire width of $300 \mathrm{~nm}$, this is near the lower limit of the patterning capa-
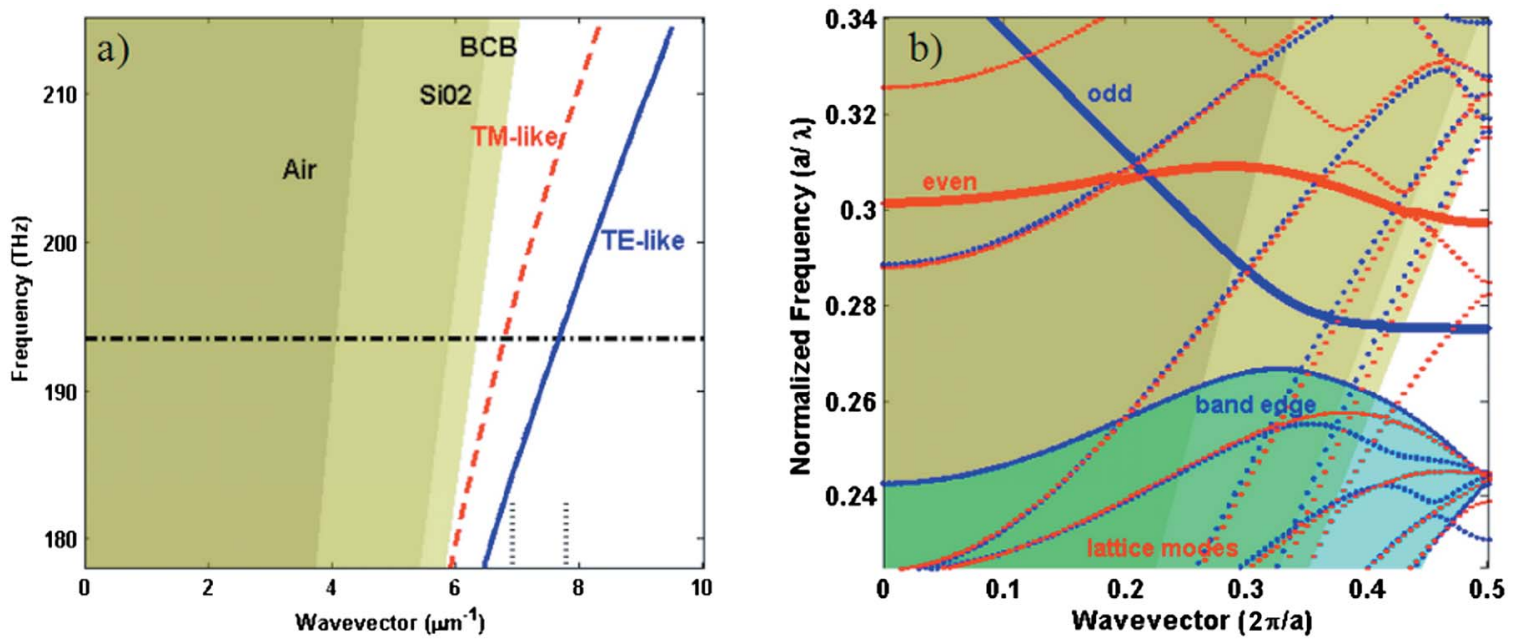

FIG. 2. (Color online) Dispersion relations. (a) The TE-like (solid line) and TM-like (dashed line) modes of a $220 \times 300 \mathrm{~nm}^{2} \mathrm{Si}_{\mathrm{i}} \mathrm{waveguide}$ with a $\mathrm{SiO} \mathrm{O}_{2}$ substrate, and BCB superstrate (see inset to Fig. 3). The light cones of the various cladding materials, air, $\mathrm{SiO}_{2}$, and $\mathrm{BCB}$ are denoted by shading. The vertical dotted lines mark the range of lithographic tuning in the experiment. The horizontal line marks the peak gain wavelength of our QWs. (b) The 3D PhC bandstructure calculated using a guided mode expansion for an InP membrane with a BCB substrate and air superstrate (with air holes). The thick line (marked odd) corresponds to the odd TE-like defect mode and the thick line (marked even) the even TE-like defect mode. The TE-like band edge modes are shaded. The TM-like lattice modes are plotted as dotted lines. The period is a=435 $\mathrm{nm}$, hole radii $\mathrm{r}=0.3 \mathrm{a}$ and the height of the InP is $255 \mathrm{~nm}$, the supercell is $5 \sqrt{ }(3)$ a wide. 

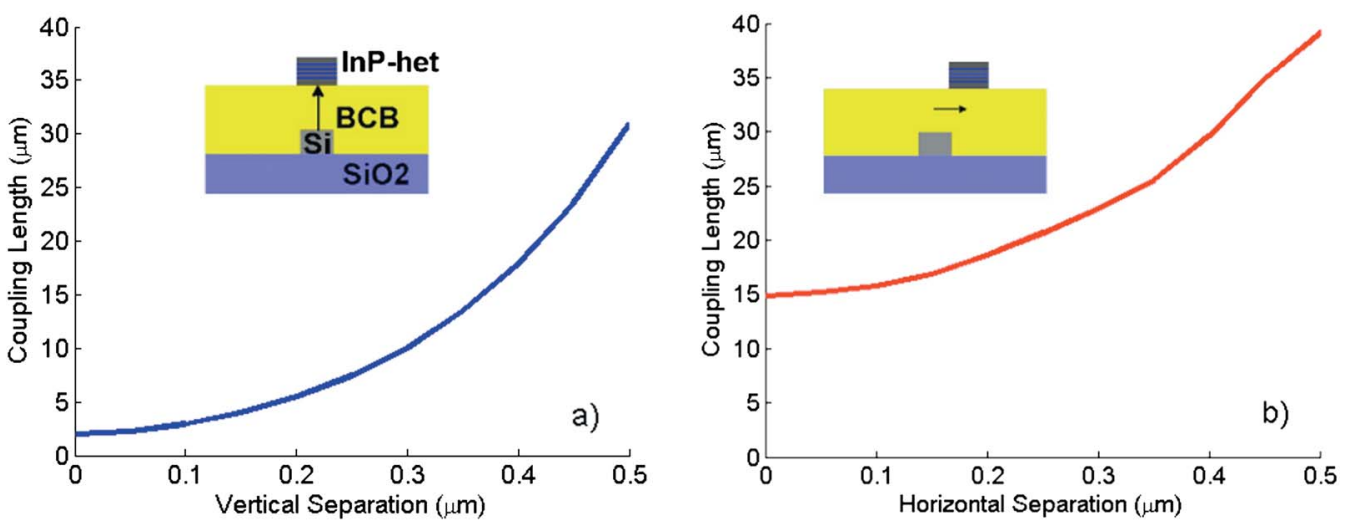

FIG. 3. (Color online) The variation in coupling length of an InP-based $\left(255 \times 360 \mathrm{~nm}^{2}\right)$ wire phase matched to a $\left(220 \times 300 \mathrm{~nm}^{2}\right) \mathrm{Si}$ wire as a function of the (a) vertical and (b) lateral offset between the guides at a constant vertical offset of $400 \mathrm{~nm}$ for $\lambda=1.55 \mu \mathrm{m}$.

bilities of the DUV process. The dispersion of a $300 \mathrm{~nm}$ wide waveguide is plotted in Fig. 2(a). One TE-like and one TMlike mode are guided. For a $\mathrm{PhC}$ period of $\mathrm{a}=435 \mathrm{~nm}$ and $\mathrm{r}=0.3 \mathrm{a}$, the mode edge of the InP W1 defect waveguide occurs at $1.55 \mu \mathrm{m}$, and has an effective index, $\mathrm{n}_{\text {eff }}$ of 1.78 . At the same wavelength a $300 \mathrm{~nm} \mathrm{Si}$ guide sitting on a $\mathrm{SiO}_{2}$ substrate with $\mathrm{BCB}$ superstrate has an $\mathrm{n}_{\text {eff }}$ of 1.91. By fabricating a series of structures with different periods we can tune the modes of the coupled waveguides and investigate different coupling regimes at or close to a phase matched condition.

A couple of drawbacks naturally exist. First heterogeneous integration creates a vertical asymmetry, which permits polarization mixing. Odd (even) TE-like modes couple with odd (even) TM-like modes, respectively, and any lateral misalignment removes the symmetry entirely. Second, the relatively high refractive index of the $\mathrm{BCB}$ (1.54) cladding, as compared to $\mathrm{SiO}_{2}$ (1.45) or air (1) reduces the optical confinement [as shaded in Figs. 2(a) and 2(b)]. Although the layer thickness of the $\mathrm{BCB}$ is in the range of hundreds of nanometers its refractive index fixes the limit to lossless guiding in this system. Modes folded above the light line of the $\mathrm{BCB}$ are coupled to the radiation continuum giving a bandwidth limit to the devices. Both $\mathrm{PhC}$ and SOI waveguides offer strong modal confinement and so we need to be able to appreciate the tolerance limits of our alignment techniques, if we want to transfer energy between the two waveguides.

With the aim of understanding the alignment tolerance we briefly investigate, using a numerical calculation, the coupling between two index matched waveguide modes and its dependence upon vertical and lateral misalignment. This study neglects the periodicity of our experimental waveguide system. Using a finite difference fully vectorial mode solver, we first find the parameters which phase match the fundamental mode of a $220 \times 300 \mathrm{~nm}^{2} \mathrm{SOI}$ waveguide $\left(\mathrm{SiO}_{2}\right.$ substrate and BCB superstrate) with that of a $255 \mathrm{~nm}$ high $\mathrm{InP} /$ InGaAs QW waveguide (BCB substrate and air superstrate), each having an effective index of $\mathrm{n}_{\text {eff }}=1.91$. The coupling length increases exponentially with separation in both $\mathrm{z}$ and $y$ directions, as expected. It can be seen [from the data presented in Fig. 3(b)] that tight lateral positioning control is required on a scale of about $100 \mathrm{~nm}$ (which is only possible using e-beam alignment), $100 \mathrm{~nm}$ misalignment would give an increase in the coupling length of about $15 \%$.

\section{FABRICATION}

\section{A. Si waveguide definition and InP wafer growth}

The SOI waveguides are patterned using $193 \mathrm{~nm}$ DUV lithography on a CMOS line. These are then etched with a $\mathrm{ClHBr}$ reactive ion etch (RIE). The $220 \mathrm{~nm} \mathrm{Si}$ layer is entirely etched through, leaving a $220 \times 300 \mathrm{~nm}^{2}$ waveguide on a $2 \mu \mathrm{m}$ buried oxide, with a silicon substrate. Alignment marks for subsequent processing are defined in the $\mathrm{Si}$ on the same mask layer and relative to the waveguide structures. The Si wires are tapered up to a width of $10 \mu \mathrm{m}$ for output coupling via shallow etched grating couplers. ${ }^{17}$ The InP material is grown by metal-organic chemical-vapor deposition, and contains four InGaAs/InGaAsP QWs emitting at a peak photoluminescence (PL) wavelength of $1.53 \mu \mathrm{m}$.

The silicon waveguide dimensions have been confirmed by scanning electron microscope (SEM) measurements of the width $(326 \mathrm{~nm})$, the wire width being varied deliberately across the 8 in. wafer following the exposure dose. The III-V slab thickness $(255 \mathrm{~nm})$ was measured by wet etching back the InP and QW layers and using a Dektak surface profiler.

\section{B. Bonding}

A $1.4 \times 1 \mathrm{~cm}^{2}$ SOI die is diced from an 8 in. wafer and a roughly $5 \mathrm{~mm}^{2}$ die of InP is cleaved [see Fig. 4(a), (i)]. Achieving a high quality bonded interface requires two clean surfaces. A standard RCA-1 (DI water $+\mathrm{NH}_{4} \mathrm{OH}+\mathrm{H}_{2} \mathrm{O}_{2}$, 5:1:1 at $70{ }^{\circ} \mathrm{C}$ ) clean is performed for the Si layer and the $\mathrm{InP}$ is cleaned with acetone-IPA. The BCB is diluted in an appropriate ratio of mesitylene in order to obtain the chosen spun film thickness, ${ }^{18}$ which for the samples fabricated here is $\sim 400 \mathrm{~nm}$ (top of $\mathrm{Si}$ to lower InP surface). After application of an adhesion promoter (AP3000) the BCB is spun (5000 rpm, $30 \mathrm{~s}$ ) onto the SOI, outgassed at $80{ }^{\circ} \mathrm{C}$ for several minutes to avoid the presence of bubbles that may otherwise introduce defects into the final structure. Finally the InP material is gently placed over the site of the patterned waveguides, while the sample is heated to $150{ }^{\circ} \mathrm{C}$ [see Fig. 4(a), (ii)]. In order to polymerize the $\mathrm{BCB}$ and finalize the 


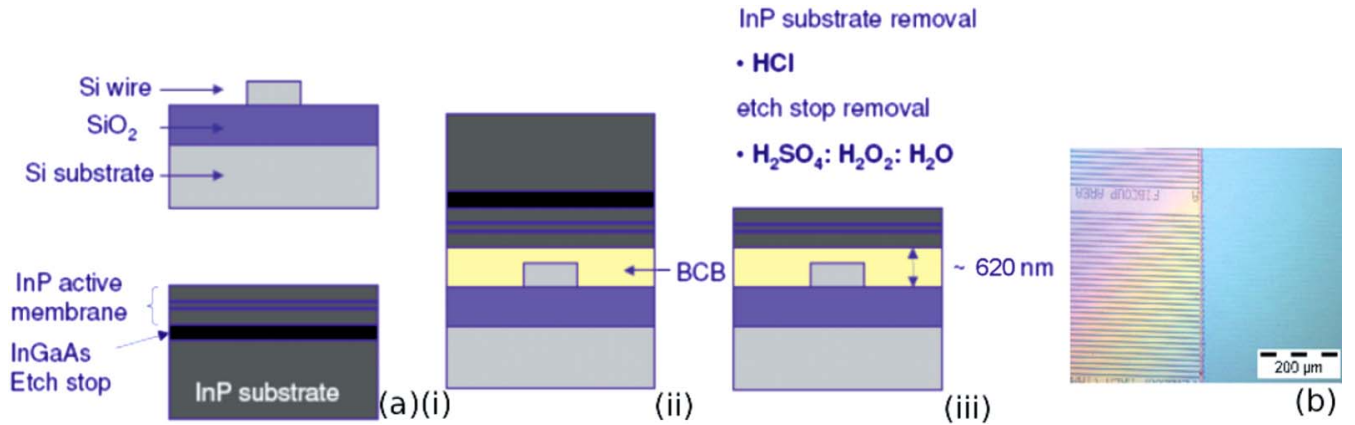

FIG. 4. (Color online) (a) Steps to achieve membrane bonded above waveguide. The two wafers are cleaned, then diluted BCB is spun onto the SOI waveguides and the InP die is positioned on top. After hard baking, the InP substrate is removed with a wet etch. (b) Optical image of SOI waveguides with bonded InP slab on the right hand side, the $\mathrm{Si}$ waveguides are just visible beneath the $\mathrm{InP}$.

bonding a hard bake is performed in a nitrogen atmosphere, for $2 \mathrm{~h}$ at $300{ }^{\circ} \mathrm{C}$. Once hard baked the InP substrate is removed by $\mathrm{HCl}$ wet etch. The $\mathrm{InGaAs}$ etch stop is removed using $\mathrm{H}_{2} \mathrm{SO}_{4}: \mathrm{H}_{2} \mathrm{O}_{2}: \mathrm{H}_{2} \mathrm{O}(3: 1: 1)$ to leave the $255 \mathrm{~nm} \mathrm{InP}$ based membrane bonded to the SOI [see Fig. 4(a), (iii)]. An optical image of the bonded material is shown in Fig. 4(b).

\section{PhC patterning and alignment}

Patterning the PhCs into the bonded $\mathrm{InP}$ material requires a two stage masking process. A $300 \mathrm{~nm} \mathrm{Si}_{3} \mathrm{~N}_{4}$ mask is deposited by plasma-enhanced chemical-vapor deposition to act as a hard mask. This layer also serves to protect the BCB and underlying silicon guides in later processing steps. On top of this we spin coat and bake a $450 \mathrm{~nm}$ polymethyl methacrylate (PMMA) mask.

To align our guides, our "semiautomatic" alignment technique requires four marks defined in the same high resolution mask layer as the $\mathrm{Si}$ waveguides. In order for the Leica EPBG5000+e-beam writer to be able to detect and recognize these marks, they must be of suitable contrast. The backscattered signal is directly related to the atomic number of the material in which the mark is defined. The abruptness of the edges of the mark is paramount to ensuring the correct measurement of the position of their center. In these thin semiconductor films, at the high electron writing energy (100 $\mathrm{keV}$ ) employed, the backscattered signal is characteristically weak and of poor contrast. To add to this difficulty the multilayer semiconductor/dielectric stack is prone to charging, as the $\mathrm{BCB}$ (and also the $\mathrm{SiO}_{2}$ ) is a very efficient insulating layer. To overcome these difficulties a wide range of mark geometries and configurations was tested. Our final choice consists in using $10 \times 10 \mu \mathrm{m}^{2}$ squares etched through the $220 \mathrm{~nm}$ silicon and the $2 \mu \mathrm{m} \mathrm{SiO}_{2}$ layers. The contrast of these CMOS compatible marks allows us to repeatably detect them and thereby align the $\mathrm{PhC}$ to the $\mathrm{Si}$ waveguides. The marks permit us to correct for the rotation, shift, and scaling between the $\mathrm{Si}$ and InP layers. The $\mathrm{PhC}$ waveguide patterns are exposed in the PMMA along with a $2 \mu \mathrm{m}$ wide moat (in the same high resolution layer).

The moat allows us to very simply discard the superfluous InP at a later processing step, avoiding the absorption that this material would otherwise cause. Following the development of the PMMA, the pattern is transferred into the $\mathrm{Si}_{3} \mathrm{~N}_{4}$ mask by a RIE using a $\mathrm{SF}_{6} / \mathrm{CHF}_{3}$ chemistry [gas ratio
10/14 SCCM (SCCM denotes cubic centimeter per minute at STP)]. To avoid reflow during the subsequent high temperatures steps the PMMA is then also removed in a RIE dry etch organic cleaning process. The $\mathrm{PhC}$ is etched into the InP layer by inductively coupled plasma (ICP)-RIE (Ref. 19) with $\mathrm{Cl}_{2} / \mathrm{BCl}_{3} \mathrm{~N}_{2}$ chemistry. The $\mathrm{Si}_{3} \mathrm{~N}_{4}$ mask is then removed by repeating the previous RIE process. We then remove the superfluous InP material. Using the alignment marks and a negative resist (maN 2410) a low resolution area is exposed, using e-beam lithography, $1 \mu \mathrm{m}$ narrower than the moat. This mesa protects the underlying $\mathrm{PhC}$ from a three step wet etching cycle using $\mathrm{HCl}$, $\mathrm{H}_{2} \mathrm{SO}_{4}: \mathrm{H}_{2} \mathrm{O}_{2}: \mathrm{H}_{2} \mathrm{O}(3: 1: 1)$ and once again $\mathrm{HCl}$, which removes the InP and the InGaAs/InGaAsP QWs. Samples of two different lengths, 30 and 200 periods, were fabricated. The technological steps as well as the optical images of the sample after each step are shown in Figs. 5 and 6.

\section{ALIGNMENT OF WAVEGUIDES}

During the InP etching step, the BCB layer was slightly thinned and actually allow us to obtain reasonably contrasted SEM images of the ends of the PhCs and the silicon waveguide layer, beneath the residual BCB, as can be seen in Fig. 7.

By fitting the waveguide position and the hole centers over a selection of guides, we can gain a statistical measure of our alignment capabilities.

To determine the position of the waveguide at the end of the $\mathrm{PhC}$ an adaptive supergaussian function (variable width, height, and center) is fitted to each transverse section of the wire, (determining the position of the guide provides the largest measurement error, WG error, as displayed in the 3rd

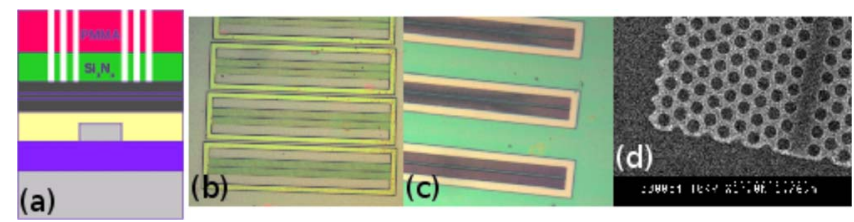

FIG. 5. (Color online) (a) The hard $\mathrm{Si}_{3} \mathrm{~N}_{4}$ mask is etched using Fluorinebased RIE through the patterned PMMA obtained after aligned electron beam lithography. (b) Optical images showing the $\mathrm{PhC}$ patterning of first the soft PMMA mask (aligned with the guide below) and (c) its subsequent transfer into the $\mathrm{Si}_{3} \mathrm{~N}_{4}$ hard mask by RIE etch. (d) An SEM image reveals the island of patterned $\mathrm{Si}_{3} \mathrm{~N}_{4}$. 


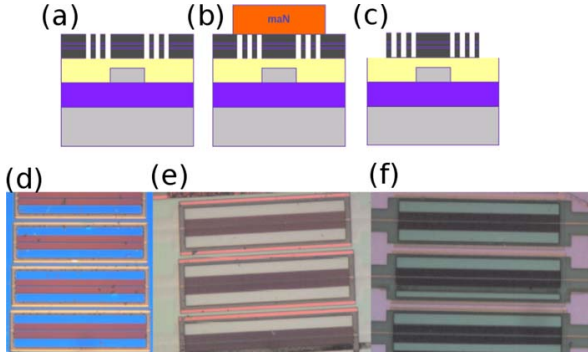

FIG. 6. (Color online) (d) The $\mathrm{Si}_{3} \mathrm{~N}_{4}$ mask (a) The InP layer is etched using inductively coupled plasma (ICP), and in the lower optical image (e) the Si waveguides become visible. The $\mathrm{Si}_{3} \mathrm{~N}_{4}$ mask is removed and a electronic negative resist maN is patterned (b) to efficiently protect the $\mathrm{PhC}$ while the superfluous InP material is removed. (c) and (f) The final structures: bonded, accurately aligned, and patterned on top of the $\mathrm{Si}$ wire waveguides.

and 5th columns of Table I). A linear fit through these center positions is extrapolated to the end of the PhC. After thresholding the $\mathrm{PhC}$ region the edges of all the shapes ( $\sim 30$ holes $)$ in the structure are detected, using a customwritten code. Each promising candidate is fitted with a circle using Taubin's method as shown in Fig. 8. The circle center coordinates are then least-squares fitted to the designed structure ( $\sim 1 \mathrm{~nm}$ error). The predicted end position of the $\mathrm{PhC}$ is then subtracted from the waveguide position, to give the alignment offset. These results are displayed in Table I.

We checked each of the nine individual longer waveguides to determine a statistical alignment accuracy. Averaging over the alignment errors for left and right hand side ends of the $\mathrm{PhC}$ waveguides gives $26 \pm 19 \mathrm{~nm}$. This accuracy is well below the $100 \mathrm{~nm}$ value which we saw (in Fig. 3) would lead to an increase in the coupling length of $15 \%$. With good control over the fabricated waveguides we now look at the optical properties of these hybrid structures, first numerically and then optically.

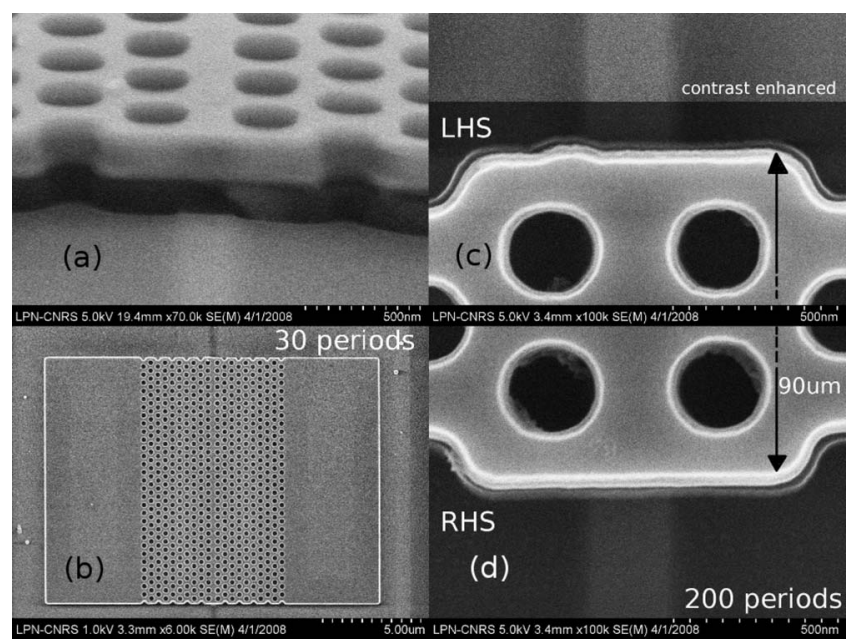

FIG. 7. (a) SEM angled view of the end of the sample showing the patterned InP layer on top of the etched $\mathrm{BCB}$, the $\mathrm{Si}$ guide is barely visible to the SEM, here we see a slightly lighter region, (b) full InP mesa containing etched $\sim 13 \mu \mathrm{m}$ "short" PhC (c) LHS of longer guide with enhanced contrast to display alignment with underlying waveguide, (d) RHS of waveguide, which is $\sim 90 \mu \mathrm{m}$ further along the waveguide.
TABLE I. The fitted alignment offset tolerances for nine $\sim 90 \mu \mathrm{m} \mathrm{PhC}$ long waveguides, along with the error in determining the waveguide position, WG error

\begin{tabular}{lccccc}
\hline \hline & \multicolumn{2}{c}{ Left hand side } & & \multicolumn{2}{c}{ Right hand side } \\
\cline { 2 - 3 } \cline { 5 - 6 } \cline { 5 - 6 } & $\begin{array}{c}\text { Alignment offset } \\
(\mathrm{nm})\end{array}$ & $\begin{array}{c}\text { WG error } \\
(\mathrm{nm})\end{array}$ & & $\begin{array}{c}\text { Alignment offset } \\
(\mathrm{nm})\end{array}$ & $\begin{array}{c}\text { WG error } \\
(\mathrm{nm})\end{array}$ \\
\hline Guide 1 & -21 & 7 & & -34 & 5 \\
Guide 2 & 16 & 8 & & 15 & 3 \\
Guide 3 & -7 & 5 & & 12 & 3 \\
Guide 4 & -32 & 3 & & 14 & 4 \\
Guide 5 & 18 & 4 & & 21 & 3 \\
Guide 6 & -57 & 4 & & -80 & 4 \\
Guide 7 & 16 & 6 & & 44 & 3 \\
Guide 8 & 3 & 4 & & 33 & 3 \\
Guide 9 & -18 & 4 & & 28 & 3 \\
\hline \hline
\end{tabular}

\section{MODELING, OPTICAL CHARACTERIZATION, AND DISCUSSION}

\section{A. Numerical simulations}

In order to study numerically the coupled system we have chosen a 3D finite difference time domain (FDTD) (full-vectorial) modeling tool. ${ }^{20}$ We consider the system to be entirely passive, i.e., we do not include the absorption/gain of the QWs. In the propagation direction $\mathrm{x}$, the InP PhC has discrete translation symmetry whereas the $\mathrm{Si}$ wire has continuous translation symmetry, so the coupled system adopts the period, a, of the $\mathrm{PhC}$ waveguide. We, therefore, create a supercell and apply Bloch boundary conditions in the $\mathrm{x}$ direction and absorbing boundary conditions in the lateral $(\mathrm{y})$ and vertical (z) directions. We can (given our high, measured alignment accuracy, which is approximately equal to one FDTD grid step) apply an odd (or even) symmetry plane at $\mathrm{y}=0$.

In Fig. 9(a) the coupled bandstructure is presented, calculated for an InP PhC W1 waveguide (period, a $=455 \mathrm{~nm}$ )

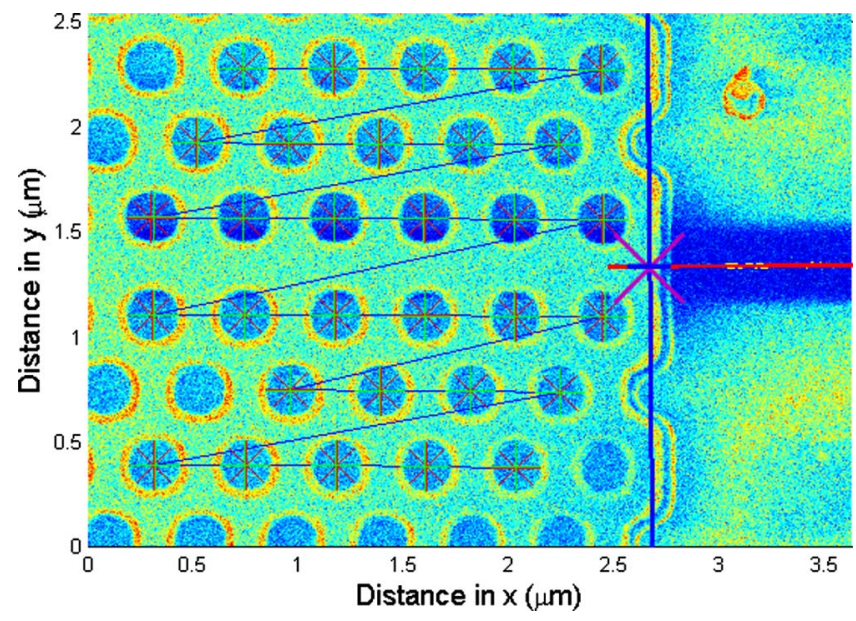

FIG. 8. (Color online) Fitted data for RHS of 4th waveguide, showing the located hole centers $(+$ crosses) and the fitted lattice (* asterisks joined by continuous zig-zagging line). The cross $(\times @ 2.669,1.324)$ shows the predicted end of the PhC waveguide. The waveguide center is shown as a noisy line and the linear fit (overlaid) predicts the intersection between the center of the waveguide and the $\mathrm{PhC}$ facet at the position marked by the darker cross (+@2.669,1.338). 


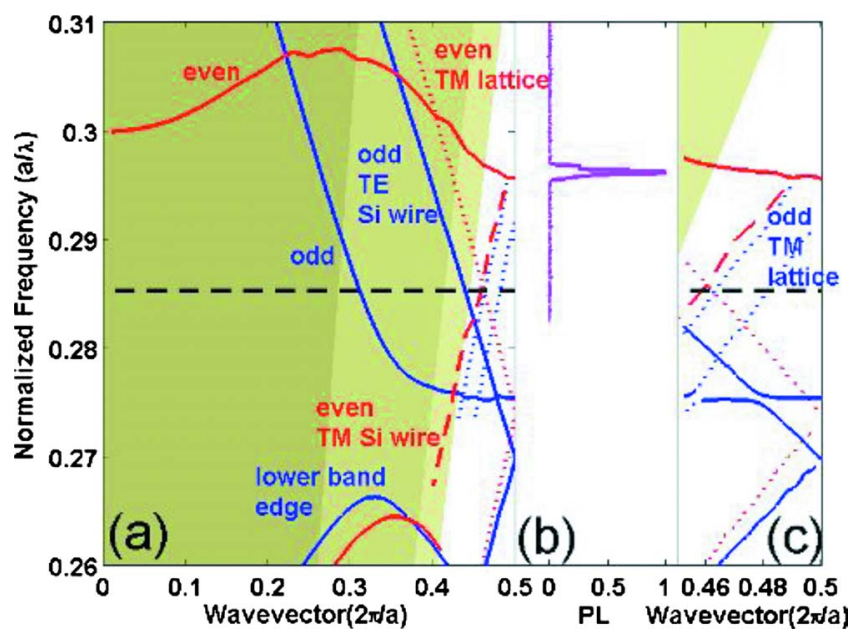

FIG. 9. (Color online) (a) Bandstructure for InP W1-Si wire (326 nm wide) coupled system, for a period, $\mathrm{a}=455 \mathrm{~nm}, \mathrm{r} / \mathrm{a}=0.3$, and a vertical separation of $400 \mathrm{~nm}$ (BCB layer). The ripple seen in the band structure is mainly due to coupling with lower Q modes. (b) The PL spectrum, coupled out via the $\mathrm{Si}$ wire, showing a single peak whose spectral position is consistent with the intersection with the even TE-like W1 mode. (c) Zooming in to the region of $\mathrm{k}$-space close to the $\mathrm{K}$ point, we can observe at $\left(\mathrm{k}_{\mathrm{x}}=0.475 \times 2 \pi / \mathrm{a}\right.$, u $=0.275$ ) an anticrossing caused by the codirectional coupling between the odd TE-like W1 mode and the odd TE-like Si wire mode.

coupled to a $220 \times 326 \mathrm{~nm}^{2}$ wide $\mathrm{Si}$ wire, situated $400 \mathrm{~nm}$ below the $\mathrm{PhC}$ waveguide. The space between the two guides is filled with $\mathrm{BCB}$. Compared to the $\mathrm{PhC}$ bandstructure represented in Fig. 2(b), we observe in Fig. 9(a) two additional lines representing the TE-like and TM-like Si wire modes, from Fig. 2(a).

One of the consequences of the $\mathrm{PhC}$ patterning is that the light emission is expected to be enhanced where the bands become flat, i.e., as $\mathrm{v}_{\mathrm{g}}$ approaches zero. For the $\mathrm{PhC}$ W1 modes this is the case at the edge of the Brillouin zone for a wave vector $\mathrm{k}_{\mathrm{x}}=\pi / \mathrm{a}$ (TE-like even mode at $\mathrm{u}=0.295$ and TE-like odd mode at $u=0.275$ on Fig. 9). The coupling between the $\mathrm{PhC}$ and the wire locally modifies the dispersion of their coupled supermodes. Even for small distances between the two waveguide layers the modal couplings are not very apparent in the large scale plot of Fig. 9(a). In order to highlight the coupling points we replot $\mathrm{k}_{\mathrm{x}}$ over a narrow range between $0.45-0.5 \times 2 \pi / \mathrm{a}$ [see Fig. 9(c)]. In the coupled system we observe at $\left(\mathrm{k}_{\mathrm{x}}=0.475 \times 2 \pi / \mathrm{a}\right.$, u $=0.275)$ an anticrossing caused by the codirectional coupling between the odd TE-like W1 mode and the odd TE-like Si wire mode [see Fig. 9(c)]. At higher frequency the even TElike W1 mode intersects the even TM-like Si wire mode, very close to the mode edge $\left(\mathrm{k}_{\mathrm{x}}=\pi / \mathrm{a}, \mathrm{u}=0.295\right)$. The periodic patterning also causes the Si mode to fold at the edge of the Brillouin zone [at $\left(\mathrm{k}_{\mathrm{x}}=\pi / \mathrm{a}, \mathrm{u}=0.27\right)$ on Fig. 9(c)]. This causes a distributed Bragg-type feedback of the light in the $\mathrm{Si}$ wire which results in a low $\mathrm{v}_{\mathrm{g}}$ point.

In Fig. 10(a) we plot the coupled bandstructure for a W1 with period, $\mathrm{a}=405 \mathrm{~nm}$, coupled in the same manner to a $220 \times 326 \mathrm{~nm}^{2}$ wide Si wire. The shift in period allows us to move these lower frequency features toward the emission bandwidth of the QWs.

We then plot the spectral positions of these various modes for fixed wave vector, $\mathrm{k}_{\mathrm{x}}=\pi / \mathrm{a}$ as a function of the

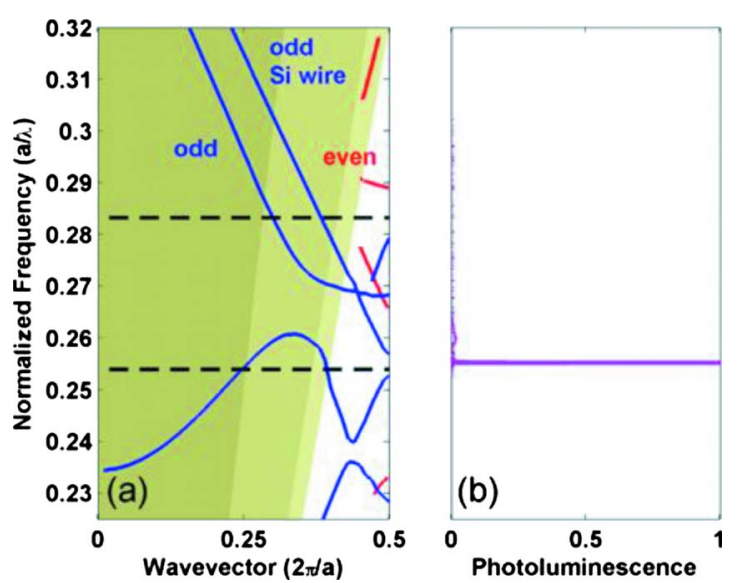

FIG. 10. (Color online) (a) Band structure for InP W1-Si wire (326 nm wide) coupled system, for period $\mathrm{a}=405 \mathrm{~nm}, \mathrm{r} / \mathrm{a}=0.3$, and a vertical separation of $400 \mathrm{~nm}$ (BCB layer). (b) The PL spectrum, coupled out via the $\mathrm{Si}$ wire. As the emission is dominated by odd modes, here we only plot the even bands in the region $\mathrm{k}_{\mathrm{x}}=0.45-0.5 \times 2 \pi / \mathrm{a}$. The dashed black lines denote the emission bandwidth of our QWs.

lattice constant of the PhC (see Fig. 11). On top of this data we plot the measurements from the optical experiments and compare these to the simulation.

\section{B. Optical characterization and discussion}

The fabricated samples are explored at room temperature as depicted in Fig. 1 by measuring the light emitted under optical pumping. The samples are surface pumped using either a $10 \times$ or a $50 \times$ IR long working distance objective. The laser source used for pumping is a Ti:Sa laser operating at a repetition rate of $80 \mathrm{MHz}$ providing $100 \mathrm{fs}$ pump pulses at $810 \mathrm{~nm}$. This near infrared (NIR) pump is absorbed by each of the semiconductor materials, the $\mathrm{Si}$, InP, and InGaAs QWs and their barriers of InGaAsP. The light emission from the III-V PhC is coupled to the $\mathrm{Si}$ wire, guided along $3 \mathrm{~mm}$ to the shallow etched TE grating couplers and diffracted into a single mode optical fiber (SMF). The SMF is angled at $10^{\circ}$ with respect to the surface normal for optimal coupling at

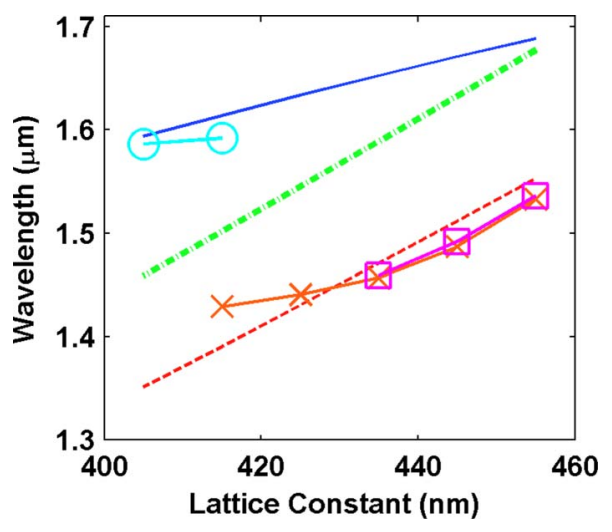

FIG. 11. (Color online) The spectral positions of the various bands at the $\mathrm{K}$ point, $\mathrm{k}_{\mathrm{x}}=\pi / \mathrm{a}$ as a function of $\mathrm{PhC}$ period. The top solid line represents the odd TE-like Si wire mode. The middle dot-dashed line denotes the odd TE-like W1 mode. The lower dashed line represents the even TE-like W1 mode. The crosses are surface detected PL. The PL extracted via the Si waveguide from the even mode is marked by squares and the circles correspond to the Si Bragg mode edge. 
$1.55 \mu \mathrm{m}$. The emission is analyzed using a spectrometer equipped with a cooled InGaAs photodiode array. Simultaneously, we detect the light emitted by the surface of the $\mathrm{PhC}$. First, we observe the PL spectra of the samples in order to identify the wavelengths where the emission is enhanced by the low $\mathrm{v}_{\mathrm{g}}$ modes of interest. The results of the measurements are shown in the central panel of Figs. 9(b) and 10(b) and their peak wavelengths are summarized in Fig. 11.

In Fig. 11, the wavelength of the observed resonances are plotted as a function of the lattice constant of the $\mathrm{PhC}$ for each sample in the range $a=405-455 \mathrm{~nm}$ in steps of $10 \mathrm{~nm}$ and for a fixed r/a ratio of 0.3 . The cyan circles on the left shows the measured resonance and corresponds to the odd TE-like Si wire mode folding, detected via the guide [the PL data are also represented in Fig. 10(b)]. The orange crosses at 1430 and $1440 \mathrm{~nm}$ are only observed in surface measurement, because these wavelengths fall out with the coupling bandwidth of the grating couplers. The magenta squares show the emission channelled via the Si wire and into the SMF.

Both the odd TE-like (green dot-dashed line) and the even TE-like (red dashed line) PhC modes shift to longer wavelength with increasing period; their dependence upon period show similar gradients. The behavior is different for the spectral position of the folding of the Si wire mode (blue line). The slope of the curve has a shallower gradient because it obeys the relationship $\lambda=2 n_{\text {effSi }} \times$ a where $n_{\text {effSi }}$ is the effective index of the waveguide mode.

We can see in the central panel of Fig. 9, an enhanced PL emission at about the same frequency as the even TE-like $\mathrm{PhC}$ mode $(\mathrm{u}=0.295)$. In order to collect the emitted light efficiently via the $\mathrm{Si}$ wire we would also expect to be close to a phase matched condition. This even TE-like PhC mode (red solid line on Fig. 9) intersects the even TM-like Si wire mode (dashed red line on Fig. 9), and due to their shared lateral symmetry and the lack of vertical symmetry they can couple. In Fig. 10(b) we see that the PL spectrum, coupled out via the $\mathrm{Si}$ wire, shows a single peak whose spectral position is consistent with the $\mathrm{K}$ point intersection of the odd TE-like Si wire mode. This is a Bragg effect imposed upon the $\mathrm{Si}$ wire modes. This large spectral feature is observed where the $\mathrm{Si}$ wire mode is folded by periodicity of the $\mathrm{PhC}$ at $\left(\mathrm{k}_{\mathrm{x}}=\pi / \mathrm{a}, \mathrm{u}=0.255\right)$. Here, the coupling is self-explanatory as the mode belongs to the $\mathrm{Si}$ wire system.

At $\left(\mathrm{k}_{\mathrm{x}}=0.47 \times 2 \pi / \mathrm{a}, \mathrm{u}=0.275\right)$ in Fig. $9(\mathrm{a})$, we notice that the flat odd TE-like mode of the $\mathrm{PhC}$ is intersected by the Si odd TE-like mode which should result in a codirectional coupling. However, in the experimental data in Fig. 11 $(425,435$, and $445 \mathrm{~nm})$, no resonances were observed probably due to losses incurred; we merely observe a broadband PL spectrum. This necessitates a deeper study and will be reported in a more exhaustive theoretical paper dealing with the coupling between wire and $\mathrm{PhC}$ defect waveguides. ${ }^{21}$

For both even TE-like PhC mode and the odd TE-like $\mathrm{Si}$ wire Bragg modes, we observe characteristic lasing behavior. The laser emission captured by the $\mathrm{Si}$ waveguide for a $=455 \mathrm{~nm}, 200$ periods $(91 \mu \mathrm{m})$ long $\mathrm{PhC}$ waveguide, is plotted in Fig. 12 as a function of the pump power on a $\log$-log scale. We obtain a classical S-shaped curve attesting

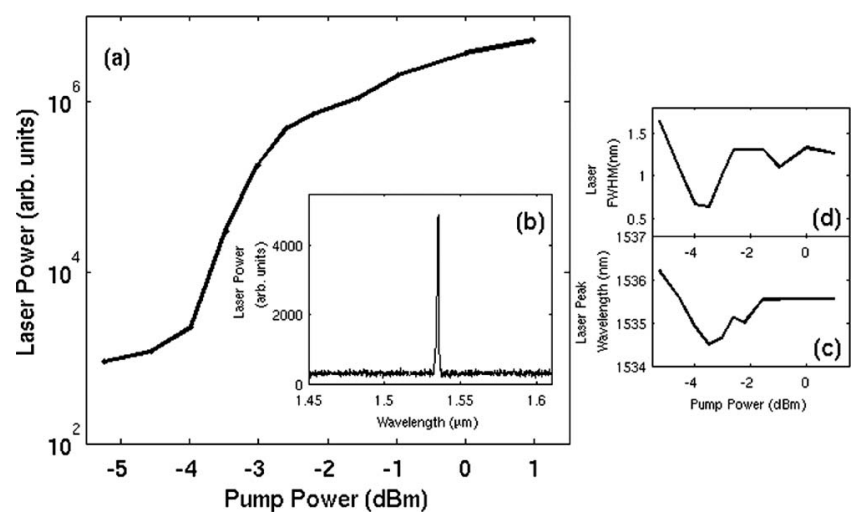

FIG. 12. (a) Laser emission power captured by the $\mathrm{Si}$ wire as a function of the pump power in log-log scale (b) Inset: laser spectrum at $1 \mathrm{~mW}$ pump power. (c) Laser peak wavelength as a function of pump power (d) Laser FWHM as a function of pump power.

to the transition from a spontaneous to a stimulated emission regime when the incident pump power is greater than 0.4 $\mathrm{mW}(5 \mathrm{pJ}$ at $80 \mathrm{MHz})$. The laser peak occurs at $1.54 \mu \mathrm{m}$ as shown in the inset of Fig. 12. As the pump power increases both the laser peak wavelength and the emission full width at half maximum (FWHM) initially decrease before increasing back up to a constant value with further increase in pump power, which is evidence for carrier clamping above laser threshold. The maximum output power coupled to the SMF is $1.2 \mathrm{fJ}$ (equivalent to $100 \mathrm{nW}$ average power). Note that similar results are also obtained with shorter $\mathrm{PhC}$ waveguides (30 periods long $\sim 13 \mu \mathrm{m}$ ).

\section{CONCLUSIONS}

We have fabricated a hybrid structure composed of active InP-based $\mathrm{PhC}$ defect waveguides coupled to SOI wires using an adhesive bonding technique. Detection of electron beam alignment marks has allowed the registration between the $\mathrm{PhC}$ W1 defect waveguide and the Si wire waveguide to be controlled to better than $30 \mathrm{~nm}$.

The light emitted under optical pumping was spectrally analyzed as a function of the lattice constant and the optical pump power. 3D FDTD numerical simulations help us identify the origin of each of the observed emission peaks as low $\mathrm{v}_{\mathrm{g}}$ modes in the bandstructures of the coupled guides. Laser operation was observed at room temperature due to the enhanced light-matter interaction provided by the low group velocity modes of the system. We show that the laser emission is coupled to the subjacent SOI waveguides. Wavelength of operation ranged from 1430 to $1595 \mathrm{~nm}$ depending on the value of the lattice constant. The laser with a period a $=455 \mathrm{~nm}$ exhibited a threshold optical pump power of 0.4 $\mathrm{mW}$ and was observed to attain an average output power of $100 \mathrm{nW}$.

In the present work laser emission is obtained under pulsed excitation. Indeed high index contrast BCB bonded membranes exhibit poor thermal dissipation. Technologically the main challenges are to achieve adequate thermal dissipation to enable $\mathrm{CW}$ laser operation. The choice of bonding 
material has been shown to be a factor ${ }^{9,10}$ in this respect. Accurate control of the thickness of the bonding layer is also critical in attaining repeatable coupling.

Several improvements can be conceived to ameliorate the system. One obvious modification has already been implemented which consists of using the optical modes in the system to pump the sample via the Si wire waveguide so that the pump is absorbed more efficiently and uniformly. This constitutes an alternative to the ambitious goal of electrical injection of these lasers; a topic requiring further study to find configurations for contacting without inducing high optical losses. The PhC defect can also be structured to remove higher order modes and also marginally increase the group velocity of the low $\mathrm{v}_{\mathrm{g}}$ modes. The coupled bandstructure (for the same $400 \mathrm{~nm}$ vertical offset) is much more perturbed for a W0.65 waveguide coupled to a Si wire than for the W1 studied here. These advances are reported in Ref. 22. It would certainly be very interesting to be able to sweep the dispersion of the $\mathrm{Si}$ wire mode through the regimes which contra and codirectional couple to the PhC modes. Both lasing and amplification regimes need to be more thoroughly investigated. This periodic high refractive index contrast environment can provide tight confinement (low optical mode volume) in nanocavities and our high precision alignment technique permits us to envisage ultrasmall active components coupled to passive waveguides.

\section{ACKNOWLEDGMENTS}

The SOI waveguides were fabricated at IMEC as part of the ePIXnet Silicon Platform (now ePIXfab). The authors would like to acknowledge the ICT FP7 European Project HISTORIC, COST MP0702 and ANR jeunes chercheurs PROWOC French national project for funding. We would like to thank Pieter Dumon for help with the Si mask.
${ }^{1}$ B. Jalali and S. Fathpour, J. Lightwave Technol. 24, 4600 (2006).

${ }^{2}$ H. Park, A. Fang, S. Kodama, and J. Bowers, Opt. Express 13, 9460 (2005).

${ }^{3}$ J. V. Campenhout, P. R. Romeo, P. Regreny, C. Seassal, D. V. Thourhout, S. Verstuyft, L. Di Cioccio, J.-M. Fedeli, C. Lagahe, and R. Baets, Opt. Express 15, 6744 (2007).

${ }^{4}$ D. Englund, H. Altug, B. Ellis, and J. Vuckovic, Laser Photonics Rev. 2, 264 (2008).

${ }^{5}$ F. Raineri, C. Cojocaru, P. Monnier, A. Levenson, R. Raj, C. Seassal, X. Letartre, and P. Viktorovitch, Appl. Phys. Lett. 85, 1880 (2004).

${ }^{6}$ A. Berrier, Y. Shi, J. Siegert, S. Marcinkevicius, S. He, and S. Anand, J. Vac. Sci. Technol. B 27, 1969 (2009).

${ }^{7}$ K. Nozaki, H. Watanabe, and T. Baba, Appl. Phys. Lett. 92, 021108 (2008).

${ }^{8}$ L. Lu, A. Mock, M. Bagheri, E. H. Hwang, J. O’Brien, and P. D. Dapkus, Opt. Express 16, 17342 (2008).

${ }^{9}$ G. Vecchi, F. Raineri, I. Sagnes, K.-H. Lee, S. Guilet, L. Le Gratiet, A. Talneau, A. Levenson, R. Raj, F. Van Laere, G. Roelkens, D. Van Thourhout, and R. Baets, Opt. Express 15, 1254 (2007).

${ }^{10}$ G. Vecchi, F. Raineri, I. Sagnes, A. Yacomotti, P. Monnier, T. J. Karle, K.-H. Lee, R. Braive, L. Le Gratiet, S. Guilet, G. Beaudoin, A. Taneau, S. Bouchoule, A. Levenson, and R. Raj, Opt. Express 15, 7551 (2007).

${ }^{11}$ F. Raineri, A. M. Yacomotti, T. J. Karle, R. Hostein, R. Braive, A. Beveratos, I. Sagnes, and R. Raj, Opt. Express 17, 3165 (2009).

${ }^{12}$ G. Roelkens, J. Brouckaert, D. V. Thourhout, R. Baets, R. Notzel, and M. Smit, J. Electrochem. Soc. 153, G1015 (2006).

${ }^{13}$ K. Sakoda, Optical Properties of Photonic Crystals (Springer-Verlag, Berlin, 2001).

${ }^{14}$ L. C. Andreani and D. Gerace, Phys. Rev. B 73, 235114 (2006).

${ }^{15}$ K. Kiyota, T. Kise, N. Yokouchi, T. Ide, and T. Baba, Appl. Phys. Lett. 88, 201904 (2006).

${ }^{16}$ P. E. Barclay, K. Srinivasan, M. Borselli, and O. Painter, Opt. Lett. 29, 697 (2004).

${ }^{17}$ D. Taillaert, F. Van Laere, M. Ayre, W. Bogaerts, D. Van Thourhout, P. Bienstman, and R. Baets, Jpn. J. Appl. Phys., Part 1 45, 6071 (2006).

${ }^{18}$ G. Roelkens, D. V. Thourhout, and R. Baets, Electron. Lett. 41, 561 (2005).

${ }^{19}$ K.-H. Lee, S. Guilet, G. Patriarche, I. Sagnes, and A. Talneau, J. Vac. Sci. Technol. B 26, 1326 (2008).

${ }^{20}$ www.lumerical.com

${ }^{21}$ T. J. Karle, Y. Halioua, R. Raineri, I. Sagnes, and R. Raj, "Intricacies of evanescent coupling between Silicon wire waveguides and Photonic Crystal line defect waveguides" (unpublished).

${ }^{22}$ Y. Halioua, T. J. Karle, F. Raineri, P. Monnier, I. Sagnes, G. Roelkens, D. Van Thourhout, and R. Raj, Appl. Phys. Lett. 95, 201119 (2009). 